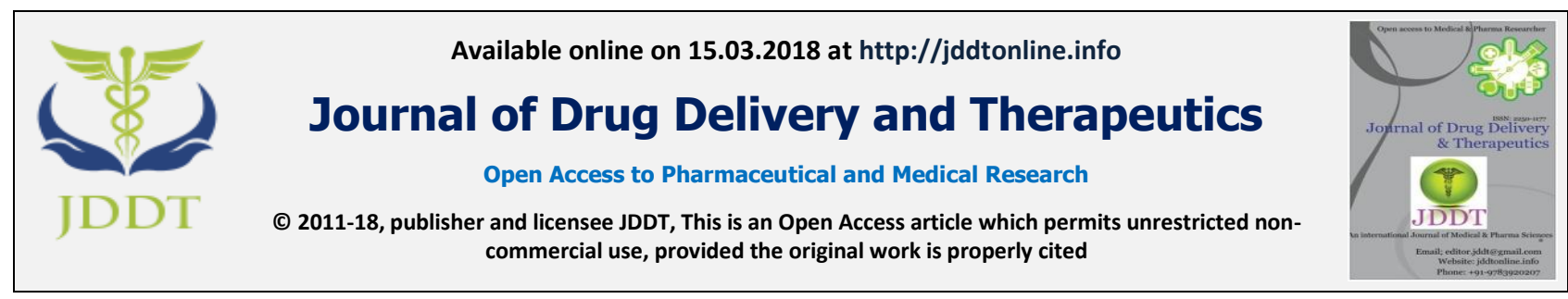

Open $\odot$ Access

Research Article

\title{
MULTIDRUG RESISTANT TUBERCULOSIS IN CHILDREN IN THE DEMOCRATIC REPUBLIC OF CONGO: FIRST EXPERIENCE WITH A SHORT TREATMENT COURSE IN A UNIVERSITY HOSPITAL
}

\author{
*Aketi Loukia ${ }^{1}$, Shiku Diayisu Joseph ${ }^{1}$, Bakoko Brian², Anshambi Nicole ${ }^{2}$, Kashongwe Zacharie ${ }^{3}$ \\ ${ }^{1}$ Department of Pediatrics, University Hospital of Kinshasa, Kinshasa, Democratic Republic of Congo \\ ${ }^{2}$ Provincial Coordination of Tuberculosis and Leprosy, Kinshasa, Democratic Republic of Congo \\ ${ }^{3}$ Department of Internal Medicine, University Hospital of Kinshasa, Kinshasa, Democratic Republic of Congo
}

\section{ABSTRACT}

Background: A short treatment course for multidrug-resistant tuberculosis (MR-TB) is not yet well codified in children in the Democratic Republic of Congo (DRC). The objective of this study was to evaluate a short MR-TB treatment course in children.

Methods: A prospective study was performed from April 2015 (corresponding to the inclusion) through April 2017 (and the later initiation time point was April 2016) in the University Clinics of Kinshasa. Enrolled children were aged 0 to 15 years. The treatment duration was in general for 9 months, with 4 months of intensive phase treatment with Kanamycin, Levofloxacin, Isoniazid, Pyrazinamide, Prothionamide, Clofazimine and Ethambutol, and 5 months of continuous phase treatment with Levofloxacin, Pyrazinamide, Clofazimine and Ethambutol. Frequencies were reported for significant results.

Results: A total of 21 children had MDR-TB (11 males and 10 females). Fifteen (71.43\%) were bacteriological confirmed cases (by Xpert/MTB), and $6(28.57 \%)$ were clinically diagnosed (MDR-TB contact with suggestive radiologic lesions); 2 patients were coinfected with HIV, 15 cases had pulmonary TB, and 6 had extrapulmonary TB. The main radiologic findings included TB cavity ( 3 cases), pleural effusion ( 5 cases), alveolar syndrome ( 8 cases), adenopathy ( 7 cases), and interstitial infiltration, fibrosis and miliary ( 2 cases each). The Ziehl control was negative before 4 months of treatment in the majority of the cases. Overall, 11 patients were cured, 7 completed the treatment, 2 died and 1 was lost to follow up.

Conclusions: MDR-TB remains a challenge in children. A more comfortable, short treatment course is feasible in children in the DRC. It is necessary to verify this observation with a larger cohort of MDR-TB patients in pediatrics.

Keywords: Multidrug-resistant tuberculosis; children; short treatment course; Africa; Kinshasa; treatment outcomes.

Article Info: Received 19 Jan, 2018; Review Completed 9 March, 2018; Accepted 10 March, 2018; Available online 15 March, 2018

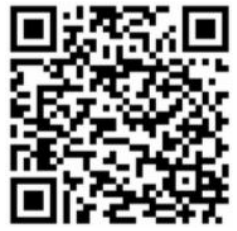

\section{Cite this article as:}

Loukia A, Joseph SD, Brian B, Nicole A, Zacharie K, Multidrug resistant tuberculosis in children in the democratic republic of Congo: first experience with a short treatment course in a university hospital, Journal of Drug Delivery and Therapeutics. 2018; 8(2):123-128

DOI: http://dx.doi.org/10.22270/jddt.v8i2.1682

*Address for Correspondence: Aketi Loukia, Department of Pediatrics, University Hospital of Kinshasa, Kinshasa, Democratic Republic of Congo

\section{INTRODUCTION}

Multidrug-resistant tuberculosis (MDR-TB) is a major health problem. It is defined as TB resistant to at least isoniazid (INH) and rifampicin (Rif). In 2016, the number of MDR-TB cases estimated worldwide was 480,000 , but the estimated number was approximately 10,4 million cases ${ }^{1}$. The Democratic Republic of the Congo (DRC), which is one of $22 \mathrm{~TB}$ high-burden countries, reported 2,790 MDR-TB cases in the same year, which represents $2.2 \%$ of new TB cases and $11 \%$ of retreated cases ${ }^{1}$. In children, the MDR-TB situation is unknown.

There exists a relationship between the community TB prevalence and the proportion of cases found in children: the higher the prevalence, the greater the proportion ${ }^{2}$. Therefore, in regards to the situation in adults, we can extrapolate that MDR-TB in children represents a serious burden for the community and national programs. Although guidelines for adults with MDR-TB are reasonably well-defined, little guidance is 
available in children for national tuberculosis programs on planning and implementing MDR-TB services ${ }^{3}$, or the guidelines focus mainly on individual patient care ${ }^{4}$, 5 . In the DRC, a significant number of children with MDR-TB are not diagnosed and treated because there are challenges related to the microbiological diagnosis, and there are no drug formulations adapted for children in the second-line regimens. This treatment entails significant adverse events, with stigma and adherence challenges, and it requires an available adult to administer the treatment in children. To improve adherence and to render more comfortable treatment, long-term MDR-TB treatment is progressively being replaced by a short-term treatment course. In the DRC in adults, the 24 months of second-line therapy have been replaced by a shorter 9 month treatment, as used in other cohorts ${ }^{6,7,8,9}$. In children, there are no guidelines and no consensus regarding shorter second-line treatment regimens. The objective of this study is to describe the clinical profile and the efficacy of a short course treatment used in children with MDR-TB in the University Clinic of Kinshasa (UCK).

\section{METHODS}

This study was a prospective study. The cohort was identified at the time of their confirmed or presumptive MDR-TB diagnosis and then followed prospectively. A cohort of children were followed until their diagnosis of MDR-TB. They were recruited from April 2015 to December 2016, the later time point of follow up was May 2017. This period corresponded with a period of intense implementation of MDR-TB guidelines in the city. The study occurred at the University hospital of Kinshasa, which is a referral hospital. It included children aged 0 to 15 years who came from the centers for TB diagnosis and treatment (CSDT), from the national TB coordination center, or from different services within the UCK. They were transferred to the Pneumology unit of the UCK pediatric department based on suspicion for TB, for contact with a known MDR-TB case, for not responding to first-line therapy, for recurrence in a child previously treated, or for treatment after a MDR-TB confirmed diagnosis.

In children, a MDR-TB case was defined as a child with proven bacteriology or Xpert-confirmed MDR-TB or who had contact with a MDR-TB patient and had suggestive clinical or radiologic lesions ${ }^{10}$. Xpert MTBRif analysis was performed at the national laboratory of National TB Program (NTP) or in the CSDT, where the test was available; the UCK does not have this tool. Due to the rarity of children with MDR-TB, we included all the MDR-TB cases registered over the study period.

For all cases, we collected a specimen depending on the age of the patient and the form of TB. All cases relied on Xpert MTB-Rif for diagnosis (Xpert MTB/Rif; Cepheid, Sunnyvale, CA, USA), and the Ziehl test for follow up.

The anamnestic parameters were collected concerning any history of TB or MDR-TB contact, BCG vaccination, number of persons in the house, number of pieces at the house, time between diagnosis and initiation of treatment, and symptoms at admission; symptoms, clinical and paraclinical signs; parameters of follow up; and outcomes. For all children, we obtained a specimen for diagnosis, either liquid of gastric aspiration for children under 6 years old or sputum for older children. The microscopy, Xpert MTB/Rif, chest $\mathrm{X}$-ray, HIV test was performed for all included children. The Lowenstein culture was realized in 12 cases.

The treatment was initiated as soon as the diagnosis was available. Treatment was conducted for 9 months. This treatment consist on 4 months of intensive phase and 5 months of continuous phase treatments. When, at the $4^{\text {th }}$ month of treatment, the microscopy test remained positive for the bacteriologic confirmed cases or when the clinical stabilization was not attend for the clinically diagnosed cases, the intensive phase was extended for 1 additional month. The continuous phase was extended for 2 additional months when the clinical recovery was not obtained at the 9 months. Patients received Kanamycin (Kn), 15 mg/kilogram (kg) 5 days by week; Levofloxacin (LFX), $8 \mathrm{mg} / \mathrm{kg}$; Prothionamide (Proth), $15 \mathrm{mg} / \mathrm{kg}$; Clofazimine (Clof), $5 \mathrm{mg} / \mathrm{kg}$; INH, $10 \mathrm{mg} / \mathrm{kg}$; Pyrazinamide, (Z) $30 \mathrm{mg} / \mathrm{kg}$; and Ethambutol (E), 20 $\mathrm{mg} / \mathrm{kg}$, for the intensive phase, and LFX, Clof, Z and E at the same dosages for the continuous phase. These drugs have been used previously in other cohorts around the world ${ }^{6,7,8}$. The treatment was given early in the morning once per day. After treating any emergency conditions, the TB treatment was followed by ambulatory care, given by an adult who received appropriate instructions. This adult could be a parent or a tutor or a nurse. However, a nurse was required for the injectable drug. There were no pediatric formulas, and the tablets were broken and/or crushed as necessary to obtain correct dosages and available presentation for administration to the children. For small children, tablets were dissolved with water before administration. Injectable drugs were given in the buttocks in the superior and external quarters.

The follow up was planned for 1 week after the initiation of treatment, in each month for the intensive phase, and every 3 months for the second phase. For the positive cases identified by Xpert MTB-Rif, the control was determined each month until there was conversion of the Ziehl test result. The objective of the first appointment was to verify the tolerance of and adherence to the treatment and to provide psychological support to the children and/or their parents. At the initial and subsequent appointments, the tolerance and adherence were verified, and an assessment of adverse effects was made. The hemogram, transaminases, creatinine, uric acid, electrocardiograph and ionogram were performed for each control, and the auditory acuity was determined. When a child missed an appointment, a nurse was sent to the home to ask about the absence and explain again the necessity of follow up.

The data were analyzed with SPSS version 20.2 software. Frequencies and medians (with confidence interval) were reported as absolute numbers and as percentages. The Fisher test was realized to compare some data.

The ethics committee of the Public Health School of the University of Kinshasa approved the study 
(ESP/CE/042B/2015). The formal consent of the parents or tutors was obtained prior to inclusion of each child in the study. The study was conducted in accordance with the standards defined by the Declaration of Helsinki. There were no conflicts of interest. The authors did not receive any funding for this study.

\section{RESULTS}

A total of 33 cases were registered at the Pneumology service of UCK; 8 were diagnosed by Xpert before their transfer to UCK and 25 had a suspicion of MDR-TB. Among the suspicious cases, 12 (48\%) were excluded because, after analysis, they were found to have other diagnoses ( 4 cases of lymphoma, 2 cases of pulmonary tumors, 3 cases of non-specific pneumonia, 2 cases of non-specific pleural effusion, and 1 case of bronchiectasis). Twenty-one cases were ultimately included in the study: $11(52.38 \%)$ were males and 10 $(47.62 \%)$ were females, resulting in a sex ratio of 1.1 . The median age was 9.6 years (minimum, 2 weeks; maximum, 15 years). Fifteen $(71.43 \%)$ cases were confirmed by Xpert MTB-Rif (between them 11 had a positive Ziehl test and 2 had a positive Lowenstein culture), and $6(28.57 \%)$ cases had MDR-TB contact and suggestive radiologic lesions. The recruitment of cases is detailed in Figure 1. [Figure 1]

The most frequent symptoms at inclusion were persistent fever (21 cases, 100\%), persistent cough (17 cases, $80.95 \%$ ), weight loss (15 cases, $71.43 \%)$, vomiting (10 cases, 47.61\%) and dyspnea (9 cases, $42.85 \%)$. Concerning the form of TB, 15 cases $(71.43 \%)$ had PTB and $6(28.57 \%)$ had EPTB (3 pleural effusion, 2 lymph node TB, and 1 meningitis TB). Concerning radiologic findings, in $9(42.85 \%)$ cases, patients had more than 1 type of lesion. There was an alveolar syndrome in 8 cases, pleural effusion in 5 cases, mediastinal lymph node involvement in 7 cases, cavities in 3 cases, interstitial infiltration in 2 cases, miliary pattern in 2 cases, fibrosis in 2 cases, and atelectasis in 1 case. Two cases had co-infection with Human Immunodeficiency Virus (HIV). All 21 cases were analyzed by the Xpert-MTB-Rif test, and 15 tested positive as Xpert MTB-Rif resistant. Table I shows the demographic and clinical results. The Fisher test showed that the Ziehl conversion was significantly associated with outcome $(p=0.001)$. [Table I]

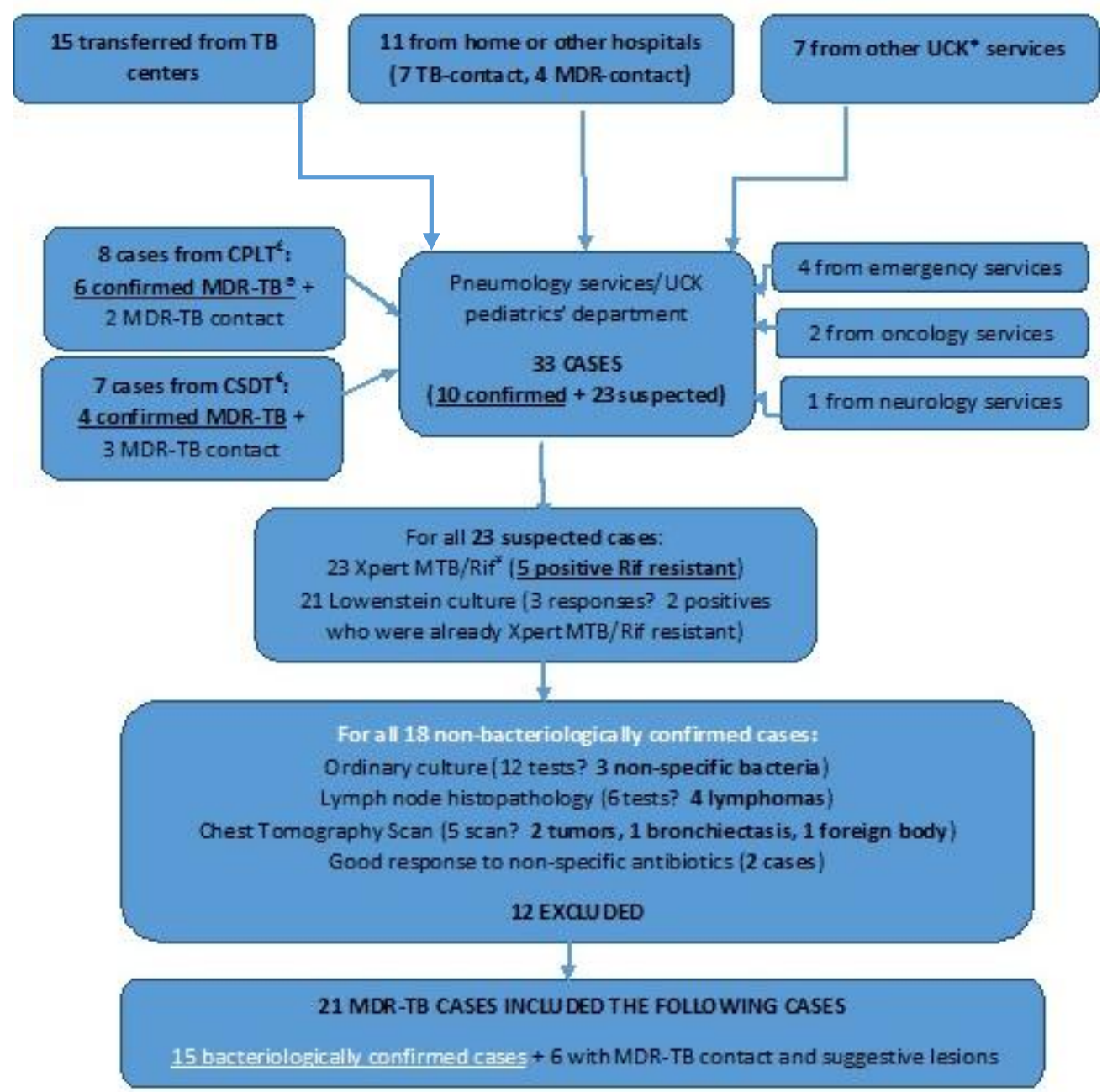

Legend: *UCK: University Clinic of Kinshasa; ${ }^{\circledR}$ CPLT: Kinshasa's provincial coordination site for TB; MDR-TB®: Multidrugresistant tuberculosis; ${ }^{\mathbf{A}} \mathrm{AFB}$ : acid-fast bacilli; ${ }^{¥}$ Rif: Rifampicin.

Figure 1: Recruitment of cases 
All patients received a 12 month short-course of secondline treatment as described above. The time between diagnosis and the initiation of this treatment ranged from 1 to 8 days.

Table 1: Distribution of demographic, clinical and paraclinical characteristics and outcomes

\begin{tabular}{|c|c|c|c|}
\hline Parameters & $\begin{array}{l}\text { Good outcome } \\
(n=18)\end{array}$ & $\begin{array}{l}\text { Poor outcome } \\
(n=3)\end{array}$ & p-value \\
\hline Age & & & 0.08 \\
\hline $0-6$ y $(n=8)$ & 5 & 3 & \\
\hline $7-15$ y $(n=13)$ & 13 & 0 & \\
\hline Sex & & & 0.24 \\
\hline Male $(n=11)$ & 8 & 3 & \\
\hline Female $(n=10)$ & 10 & 0 & \\
\hline Major symptoms* & & & 0.56 \\
\hline Persistent cough $(\mathrm{n}=17)$ & 15 & 2 & \\
\hline Weight loss $(\mathrm{n}=15)$ & 12 & 3 & \\
\hline Form of TB & & & 0.9 \\
\hline PTB $(n=15)$ & 13 & 2 & \\
\hline $\operatorname{EPTB}(n=6)$ & 5 & 1 & \\
\hline Radiologic lesions* & & & 0.9 \\
\hline Parenchymal lesions $(n=22)$ & 19 & 3 & \\
\hline Pleural or lymph impairment $(n=12)$ & 11 & 1 & \\
\hline Diagnosis & & & 0.9 \\
\hline Xpert MTB/RIF confirmed $(n=15)$ & 13 & 2 & \\
\hline MDR-TB contact with suggestive lesions $(n=6)$ & 5 & 1 & \\
\hline Ziehl test conversion $(11$ cases $)$ & & & 0.001 \\
\hline$<4$ months of treatment $(n=8)$ & 8 & 0 & \\
\hline$\geq 4$ months of treatment $(n=3)$ & 0 & 3 & \\
\hline
\end{tabular}

At follow up, the time to Ziehl test conversion was established between the 11 cases with Ziehl test positive at the initiation. The negativity on Ziehl testing appeared before or at 4 months of treatment in 8 cases (at 1 month for 3 cases, at 2 months for 2 cases, at 3 months for 2 cases, and at 4 months for 1 case), at 5 months for 1 case, at 6 months of treatment for 1 case, and at 8 months for 1 case. The median period of Ziehl conversion was 3 months (IC: 1.91-3.56 months). The radiologic follow-up showed a resolution of signs between 2 to 9.5 months for almost all cases, except for 2 cases who had chest X-ray lesions until the last month of treatment (for 1, a small pleural effusion; for the other, fibrosis). Adverse treatment effects included vomiting and stomach pain in 3 cases each (14.28\%), scratching in 2 cases $(9.52 \%)$ and urticarial rash in 1 case $(4.76 \%)$. Biologic surveillance noted high transaminase levels in 4 cases $(19.04 \%$, in 1 case more than 2 times the normal range) and high uricemia in 7 cases $(33.3 \%)$. All side effects were resolved by simple measures. For stomach pain, 2 patients received an antivomiting and 1 patient received an anti-spasmodic. For scratching and urticarial rash, patients received an antihistaminic. For patients who showed high transaminase levels, INH was stopped for 2 weeks and reintroduced progressively. For patients who had a high level of uricemia, the dose of $\mathrm{Z}$ was reduced by $5 \mathrm{mg}$ per $\mathrm{kg}$ of body weight, and for 1 case who had a persistently high level after this reduction, allopurinol was prescribed. In general, for all patients with side effects, the treatment was continued until the end, and the side effects were minimized.
Concerning outcomes, $18(85.71 \%)$ patients had a good outcome (11 were cured and 7 completed treatment), and $3(14.28 \%)$ patients had a poor outcome (2 died and 1 was lost to follow up). The first patient who died was 5 years old; he was in the first month of treatment and had a co-infection with HIV and malnutrition. The second patient was 6 years old; he died during the $7^{\text {th }}$ month of treatment, after he arrived at the hospital 2 hours before his death with dyspnea resistant to oxygen, and he remained malnourished. The Lowenstein culture of this patient did not show growth. The lost case was 4 years old; he had pneumonia with meningitis and disappeared from follow up in the fifth month of treatment. His control Ziehl test was negative at the $4^{\text {th }}$ month. The visit at home noted that he traveled with his parents.

\section{DISCUSSION}

The main objective of the study was to describe the clinical profile and to assess the efficacy of a short course treatment in children with MDR-TB in a setting where $\mathrm{TB}$ is prevalent. This study revealed that childhood MDR-TB is a reality in the DRC, especially in Kinshasa. In this series, 21 cases were diagnosed with MDR-TB; 2 had co-infections with Human Immunodeficiency Virus (HIV). Fifteen $(71.43 \%$ ) cases were confirmed by Xpert MTB-Rif, and 6 (28.57\%) cases were clinically diagnosed. Their common symptoms included persistent fever $(100 \%)$, persistent cough $(80.95 \%)$, weight loss $(71.43 \%)$, vomiting $(47.61 \%)$ and dyspnea $(42.85 \%)$; their clinical forms were PTB (15 or $71.43 \%$ ) and EPTB (6 or $28.57 \%$ ), of which 3 had pleural effusion, 1 had meningitis TB, and 
1 had lymphadenitis. The treatment outcomes were good: $85.71 \%$ had good outcome (11 cured and 7 completed treatment), and $3(14.28 \%)$ had a poor outcome ( 2 died and 1 lost was lost to follow up). The treatment was generally well-tolerated; there were 3 cases of vomiting and stomach pain, 3 skin reactions, 4 elevations of transaminases and 7 elevations of uricemia.

MDR-TB is a formidable TB infection that poses a major challenge to the global control of the disease. It is underestimated in children because of the non-specific clinical symptoms and the difficulty in confirming the diagnosis ${ }^{10}$. A definitive diagnosis of MDR-TB must include a microbiological diagnosis ${ }^{11}$. These difficulties are likely reasons why the number of MDR-TB cases was low in this study. It appears important to analyze all children with contact with MDR-TB patients and to carry out home visits to new adult MDR-TB cases to identify children with prevalent $\mathrm{TB}{ }^{12}$. It is essential to find MDR-TB cases because it has been shown that 80$90 \%$ of children with MDR-TB are usually contaminated by a close, infected adult ${ }^{13}$. Population education, especially maternal education, must be provided to improve knowledge of $\mathrm{TB}{ }^{14}$ so that all children with MDR-TB contacts will be brought to a care facility for early examination for MDR-TB.

In this study, $28.5 \%$ of patients had EPTB. It is incorrect to think that MDR-TB can present with only pulmonary involvement. In 2014, in Madrid, Santiago B et al showed an association between MDR-TB and EPTB ${ }^{15}$. In China in 2016, Qian Guo et al, in a cross-sectional study of 196 pediatric cases of mycobacterial cultureconfirmed TB, found $44.4 \%$ of cases with exclusively thoracic TB, $41.8 \%$ of cases with thoracic-extrathoracic $\mathrm{TB}$, and $13.8 \%$ of cases with exclusively extrathoracic TB ${ }^{16}$. Extrathoracic TB is more frequently encountered in children than in adults ${ }^{17}$. It is important to obtain biological specimens from any suspected anatomic sites for TB from any suspected MDR-TB case, especially in children ${ }^{16}$. The opportunity to examine any biological specimen with Xpert-MTB/Rif can facilitate diagnosis in children who have difficulty in providing sputum for analysis ${ }^{16}$. Old techniques however, like culture in Lowenstein Jensen medium, can also provide a diagnosis of MDR-TB ${ }^{18}$.

MDR-TB treatment must be initiated immediately after diagnosis to improve the outcome and reduce the risk of transmission ${ }^{12}$. In this study, treatment was delayed for several cases for 1 week because the drugs needed to be provided from another site, especially by the NTP. The university hospital does not maintain stocks of these drugs.

The WHO has proposed total treatment duration of 20 months or more, with an 8-month intensive phase, for MDR-TB patients ${ }^{19}$. However, this treatment is associated with low compliance because of the long duration, the high number of drugs to take, significant adverse effects, and stress with injectable drugs, especially for children. The low compliance has an impact on the treatment outcome ${ }^{20}$. The incidence of side effects is also important because of the immaturity of children's biological systems, and the misrelating of their early side effects can also justify the preference of short-course treatment in children. In the present study, we therefore used a short-course treatment.

Studies conducted around the world with short-course treatments ( 9 to 12 months of treatment) have shown a lower cost, a better tolerance, and a better compliance, contributing to a better efficacy $6,7,8,21,22,23$. The shorttreatment uses more efficient drugs for Mycobacterium tuberculosis $(M T)$, such as the fourth-generation quinolone Gatifloxacin, LFX, or Moxifloxacin, Clof, and a high dose of INH or Bedaquilline ${ }^{6,24,25}$.

Here, we used Clof and high doses of INH and LFX. The choice of LFX in this study was due to the early bactericidal activity of this drug in $M T$, which is slightly higher than for Gatifloxacin, and lower cardiac effects than Moxifloxacin 24, 25. In the present study, the treatment outcome was good $(85.7 \%)$, and the tolerance of treatment was acceptable because there were no major side effects.

In the present study, after stabilization of patients in the hospital, the treatment was continued by ambulatory care. For the first 6 months of treatment, patients attended the hospital or a care facility near their home for the injection of $\mathrm{KM}$; the rest of the treatment was administered at home. There are no available facilities in the University Hospital of Kinshasa to admit MDR-TB patients because there is no controlled airway or aspiration system. Thus, the treatment was administered by ambulatory care, especially after the end of the injection drug treatments. The outcomes of patients were good; only 1 patient was lost to follow up. Ambulatory care for the treatment of MDR-TB has also been used in Ethiopia with good results ${ }^{26}$.

In TB-prevalent countries, many challenges can delay the achievement of the "End TB" objectives. Among these challenges, research on MDR-TB in children is a priority ${ }^{27}$. There is a need to provide access to new MDR-TB drugs, especially for children ${ }^{28}$.

This study is the first in the DRC to use a short-course treatment in children for MDR-TB. The results showed that this treatment is feasible, efficacious, and welltolerated by children. The limit of this study is the need for a comparison with a longer one, which is not available for the moment in our context.

\section{CONCLUSION}

MDR-TB remains a challenge in children. The treatment must be initiated immediately after diagnosis to improve the outcome. A short treatment course is evaluated in children in DRC, showed a therapeutic success rate in $85.71 \%$. The treatment was comfortable in the majority of cases. More cases are needed to have a longer cohort in order to better evaluate this treatment. 


\section{REFERENCES}

[1] World Health Organization. Global tuberculosis report. Geneva; 2016.

[2] Donald P R. Childhood tuberculosis: out of control? Curr Opin Pulm Med 2002; 8:178-182.

[3] Yuen CM, Rodriguez CA, Keshavjee S, Becerra MC. Map the gap: missing children with drug-resistant tuberculosis. PHA 2015; 5(1):45-58.

[4] World Health Organization. Guidelines for the programmatic management of drug-resistant tuberculosis. Emergency update 2008. WHO/HTM/TB/2008.402. Geneva, Switzerland: WHO, 2008. http://whqlibdoc.who.int/publications/2008/9789241547581 _eng.pdf. Accessed April 2015.

[5] The Sentinel Project for Pediatric Drug-Resistant Tuberculosis. Management of multidrug-resistant tuberculosis in children: a field guide. Boston, MA, USA: The Sentinel Project for Pediatric DrugResistant Tuberculosis, 2012 http://sentinelproject.files.wordpress.com/2012/11/sentinel_project _field guide 2012. pdf. Accessed April 2015.

[6] Moodley R, Godec TR. Short-course treatment for multidrugresistant tuberculosis: the STREAM trials. Eur Respir Rev 2016; 25:29-35. DOI: 10.1183/16000617.0080-2015

[7] Trébucq A, Schwoebel V, Kuaban C, et al. Expanding shortened MDR-TB treatment: the West African experience. Int J Tuberc Lung Dis 2014; 18: Suppl. 1, S15.

[8] Aung KJM, Van Deun A, Declercq E, et al. Successful "9month Bangladesh regimen" for multidrug-resistant tuberculosis among over 500 consecutive patients. Int J Tuberc Lung Dis 2014; 18:1180-1187.

[9] Achar J, Berry C, Herboczek K, et al. Multidrug-resistant tuberculosis in child successfully treated with 9-month drug regimen. Emer Infec Dis 2015; 21(11):2105-6.

[10] World Health Organization. Guidance for national tuberculosis programmes on the management of tuberculosis in children: Second edition. Geneva; 2014.

[11] Marais BJ, Hesseling AC, Gie RP, Schaaf HS, Beyers N. The burden of childhood tuberculosis and the accuracy of communitybased surveillance data. Int J Tuberc Lung Dis 2006; 10(3):259263.

[12] Du Cros P, Swaminathan A, Bobokhojaev OI, Sharifovna ZD, Martin C, Herboczek K. Challenges and solutions to implementing drug-resistant tuberculosis programmes for children in Central Asia. PHA 2015; 5(2):99-102.

[13] Schaaf HS, Garcia-Prats AJ, Hesseling AC, Seddon JA. Managing multidrug-resistant tuberculosis in children: review of recent developments. Curr Opin Infect Dis 2014; 27 (3): 211-9. Doi: 10.1097/QCO.0000000000000062.

[14] Aketi L, Shiku DJ, Kashongwe Z, Nkabikueni G, Kayembe KP, Kayembe J-M. Maternal knowledge of tuberculosis and Bacillus Calmette Guérin vaccination in pediatric health services in Kinshasa. JTR 2017; 5:30-43. ISSN online: 2329-8448.
[15] Santiago B, Baquero-Artigao F, Mejias A, et al. Pediatric Drug-resistant Tuberculosis in Madrid: Family Matters. Pediatrc Infect Dis J. 2014; 33(4):345-350.

[16] Guo Q, Pan Y, Yang Z, et al. Epidemiology and clinical characteristics of pediatric drug-resistant tuberculosis in Chongqing, China.PLoS 2016; 11(3):e0151303. Doi:10.1371/journal.pone.0151303

[17] Alcaïs A, Fieschi C, Abel L, Casanova J-L. Tuberculosis in children and adults two distinct genetic diseases. J Exp Med 2005; 202 (2):1617-1621. PMID: 16365144

[18] Yadav S, Rawal G. Primary extrapulmonary multi-drugresistant tuberculosis in an immunocompetent child presenting with pleural effusion. Transl Pediatr 2017; 6(1):72-75.

[19] World Health Organization. Guidelines for the Programmatic Management of Drug-resistant Tuberculosis: 2011 Update. Geneva, WHO 2011.

[20] Ahuja SD, Ashkin D, Avendano M et al. Multidrug resistant pulmonary tuberculosis treatment regimens and patient outcomes: an individual patient data meta-analysis of 9,153 patients. PLoS Med 2012; 9: e1001300.

[21] Van Deun A, Maug AKJ, Salim MAH, et al. Short, highly effective, and inexpensive standardized treatment of multidrugresistant tuberculosis. Am J Respir Crit Care Med 2010; 182:684692.

[22] Piubello A, Harouna SH, Souleymane MB, et al. High cure rate with standardised short-course multidrug-resistant tuberculosis treatment in Niger: no relapses. Int J Tuberc Lung Dis 2014; 18:1188-1194.

[23] Kuaban C, Noeske J, Rieder HL, et al. High effectiveness of 12 -month regimen for MDR-TB patients in Cameroon. Int $\mathrm{J}$ Tuberc Lung Dis 2015; 19: 517-524.

[24] Stephen HG. The role of moxifloxacin in tuberculosis therapy. Eur Respir Rev 2016; 25:19-28. Doi: 10.1183/16000617.0085-2015

[25] Johnson JL, Hadad DJ, Boom WH, et al. Early and extended early bactericidal activity of levofloxacin, gatifloxacin and moxifloxacin in pulmonary tuberculosis. Int J Tuberc Dis 2006; 10:605-612

[26] Fiseha D, Kumssa H, Tefera M, Tesfaye A, Klinkenberg E, Yimer G. Ambulatory care for multidrug-resistant tuberculosis: lessons learned in Addis Ababa, Ethiopia. PHA 2014; 4(3): S37S41. doi: 10.5588/pha.14.0058

[27] Velayutham B, Nair D, Ramalingam S, Perez-Velez CM, Becerra MC, Swaminathan S. Setting priorities for a research agenda to combat drug-resistant tuberculosis in children. PHA 2015; 5(4):222-235.

[28] Cox HS, Furin JF, Mitnick CD, Daniels C, Cox V, Goemaere E. The need to accelerate access to new drugs for multidrugresistant tuberculosis. Bull World Health Organ 2015; 93:491497. doi: http://dx.doi.org/10.2471/BLT.14.138925 